\title{
La calidad como aptitud para el uso: evaluación de la exactitud posicional de los productos topográficos digitales escala 1:50,000 del INEGI
}

\author{
Héctor Mario Gómora Morales*
}

Recibido el 24 de abril de 2015; aceptado el 16 de agosto de 2015

\begin{abstract}
Throughout this article, it is intended to share a particular experience of positional accuracy evaluation, explaining its bases, design, performance and result's processing, with the aim to contribute ideas to the important and complex issue related with quality assurance of the geographic products. It is explained, too, that quality evaluation contributes to put into practice the fitness for use quality notion, which is, nowadays, relevant in the international scene.
\end{abstract}

Key words: quality, requirements, accuracy, evaluation, metadata.

\section{Resumo}

Compartilhamos ao longo deste artigo a experiência de um prova de avaliação de exatitude posicional, seu planejamento, desenho, aplicação e integração de resultados, com a finalidade de somar idéias no importante e complexo campo da qualidade em produtos geográficos. Desta forma, se explica como a avaliação da qualidade contribui para levar à prática a noção de qualidade adaptada ao uso, de grande relevância no âmbito internacional atual.

Palavras chave: qualidade, necessidades, exatitude, avaliação, metadados.

\section{Resumen}

A lo largo de este artículo se comparte la experiencia de una prueba de evaluación de exactitud posicional, sus planteamientos, diseño, aplicación e integración de resultados, con el fin de aportar ideas en el importante y complejo campo de la

* Dirección General de Geografía y Medio Ambiente, Instituto Nacional de Estadística y Geografía, Avenida Héroe de Nacozari Sur núm. 2301, Fraccionamiento Jardines del Parque, C.P. 20276, Aguascalientes, Ags., México, correo electrónico: hector.gomora@inegi.org.mx 
calidad en los productos geográficos. Asimismo se explica cómo la evaluación de la calidad contribuye a llevar a la práctica la noción de calidad de aptitud para el uso, de gran relevancia en el ámbito internacional actual.

Palabras clave: calidad, necesidades, exactitud, evaluación, metadatos.

\section{Generalidades}

El Instituto Nacional de Estadística y Geografía (INEGI) tiene entre sus objetivos suministrar a la sociedad y al Estado información de calidad, pertinente, veraz y oportuna, así como normar la generación de información estadística y geográfica en el marco del Sistema Nacional de Información Estadística y Geográfica (SNIEG). Esto último favorece un mayor intercambio de datos espaciales entre unidades productoras y usuarios, pero para que sea beneficioso se requiere que los productos geográficos incluyan información que permita usarlos con las perspectivas correctas. Proporcionar dicha información es una responsabilidad de cada Unidad del Estado que elabora productos geográficos.

En cumplimiento de su papel como productor y en apego a las buenas prácticas internacionales, el INEGI adopta la noción de calidad denominada internacionalmente fitnessfor use (aptitud para el uso), que significa determinar los niveles de confiabilidad técnica de los productos geográficos e informarlos al usuario, para que éste determine en qué medida puede usarlos para satisfacer sus requerimientos.

La Dirección General de Geografía y Medio Ambiente del INEGI ha venido realizando diferentes pruebas de evaluación de calidad, cuyos resultados se incluirán en los metadatos. Una de estas pruebas se aplicó a la información topográfica digital escala 1:50,000, que es de las más empleadas por la sociedad desde hace muchos años. A nivel mundial se reconoce que la ubicación espacial de los elementos geográficos es uno de los aspectos sobre los cuales más interesa conocer la calidad. Por ello es de los más estudiados, y se hace mediante el concepto de exactitud.

La exactitud es el grado de cercanía de un valor o conjunto de valores respecto a los valores verdaderos (o aquellos suficientemente confiables para ser aceptados como verdaderos). Llevando esta noción al ámbito de los datos espaciales, podemos hablar por ejemplo de la exactitud de los valores que definen su ubicación. A esto se le denomina generalmente exactitud posicional o exactitud de posición. En todo producto geográfico existe siempre un cierto grado de incertidumbre en la ubicación de los elementos. La evaluación y declaración de dicha incertidumbre ayuda a los usuarios a tomar conciencia de ello y superar la creencia de que exactitud significa valores perfectos, sin error alguno.

Una de las pruebas de evaluación de calidad que ha aplicado el INEGI a sus productos geográficos corresponde al tema de la exactitud posicional y se aplicó a los productos topográficos digitales escala 1:50,000. En los apartados siguientes se explican sus aspectos relevantes y resultados. 


\section{Ámbito de la evaluación}

Una evaluación de calidad debe ser delimitada con claridad en todos sus aspectos. De esta manera se sabe claramente a qué partes del producto geográfico se aplican los resultados. Empezaremos entonces por especificar el ámbito de la prueba; es decir, qué se evaluó, y a qué partes del producto.

Se evaluó la exactitud de las coordenadas planimétricas (X, Y) que definen la posición de los objetos espaciales. Esto es referido a nivel internacional como exactitud posicional horizontal. Por ello, los resultados sólo son aplicables a la planimetría, y no a la posición vertical.

Para determinar el ámbito se aplicó un principio de evaluación de la calidad que forma parte de las buenas prácticas en la materia, y al cual podemos llamar Principio de homogeneidad: evaluar y reportar por separado áreas de las cuales se asume razonablemente que tienen diferente calidad en el aspecto que se evalúa. Se tomó en cuenta que en la ubicación de los datos espaciales intervienen factores como los siguientes:

- La topografía, que según Conalton y Green (2009), tiene el mayor impacto en la exactitud posicional, por lo que una prueba de esta clase debe representar la variedad de la topografía ${ }^{1}$

- La tecnología empleada

- La exactitud de las imágenes usadas como fuente y la calidad y criterios de la extracción digital de los rasgos (en el caso de cartografía de base fotogramétrica)

- La intervención humana en distintas etapas del proceso

- El proceso de rasterización (cuando se trata de productos en formato ráster)

Teniendo presentes estos factores y el Principio de homogeneidad, el ámbito de evaluación se definió y organizó como se explica en los párrafos siguientes.

El universo lo formaron las 254 claves $^{2}$ cuya edición cartográfica se realizó en el periodo 2007-2011, del total de 2,357 que cubren el territorio nacional en escala 1:50,000. Esas 254 claves tienen en común el cambio de plataforma tecnológica empleada para la producción.

Las claves de este universo se clasificaron en tres estratos de relieve:

- Plano: predominan las planicies

- Intermedio: las planicies y las zonas accidentadas tienen extensiones más o menos similares

- Abrupto: predominan las zonas accidentadas

1 Congalton y Green, Assessing the accuracy of remotely sensed data, CRC Press, 2009, p. 27.

2 Se emplea aquí la palabra "clave" para hacer referencia a cada uno de los rectángulos de 10' x 15' en los que se divide al país para la cobertura topográfica en escala 1:50,000. 


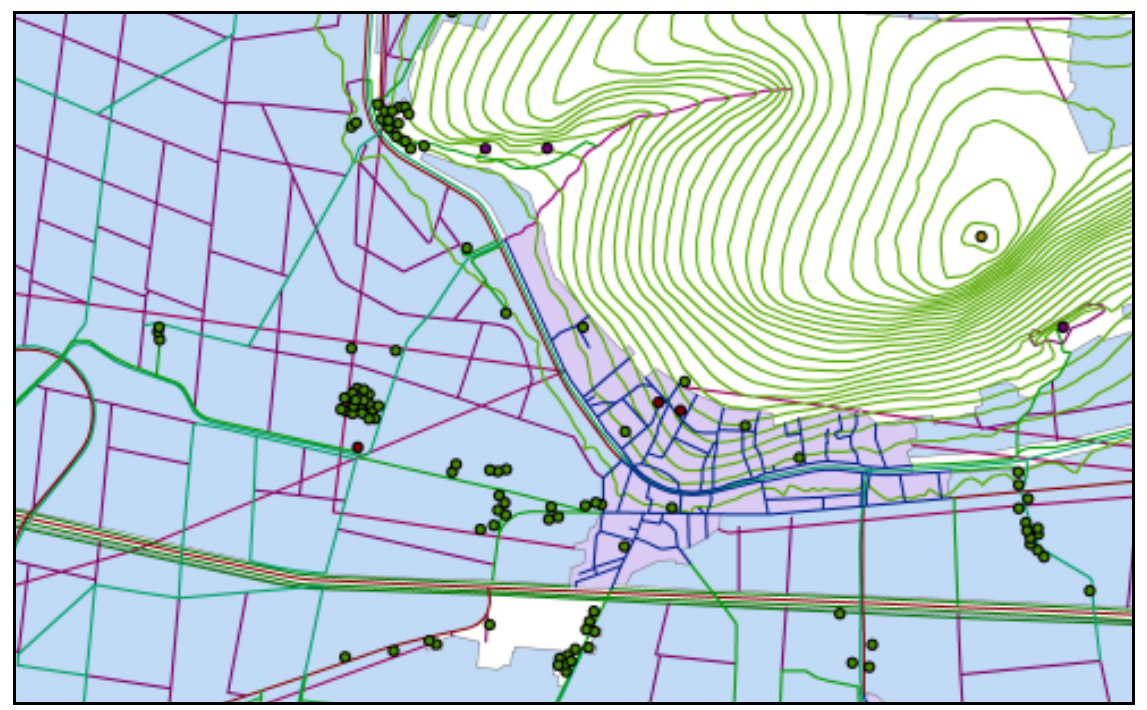

Conjunto de datos vectoriales

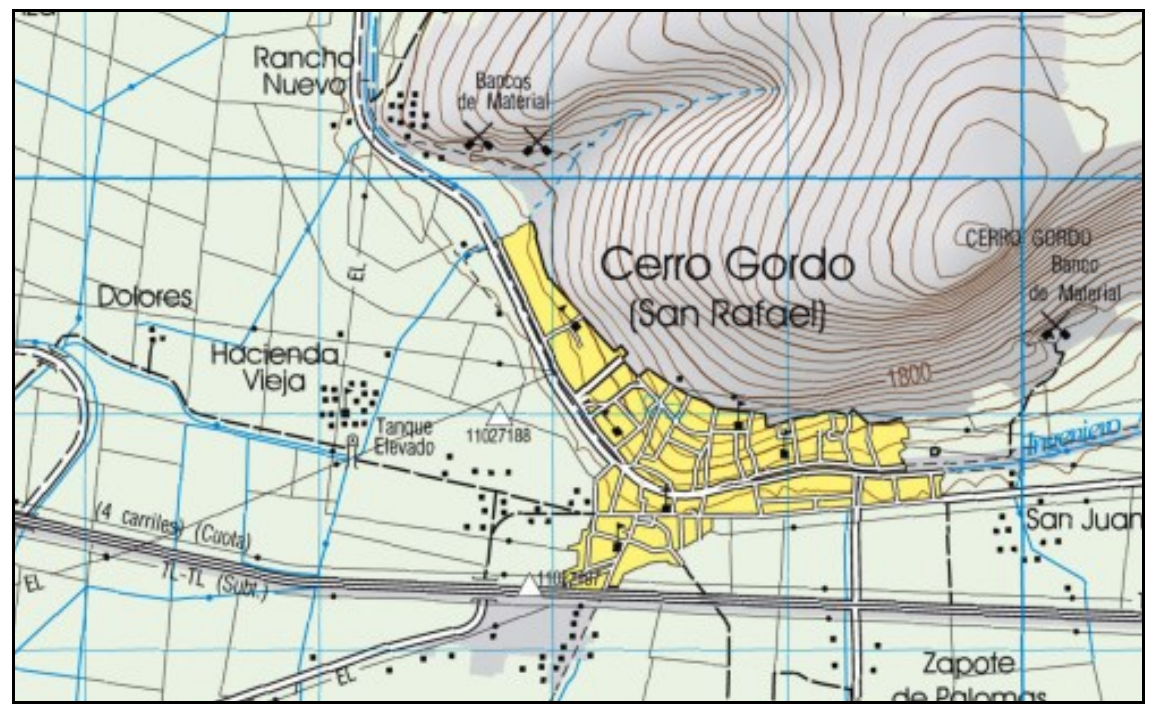

Imagen cartográfica digital

Figura 1. Productos topográficos digitales escala 1:50,000 evaluados. 
Como se mencionó antes, la prueba se concibió para los productos topográficos digitales escala 1:50,000, por lo que involucró a los conjuntos de datos vectoriales (CDV) y a la imagen cartográfica digital (ICD).

Se asumió que estos dos productos tienen diferente exactitud por las particularidades de la elaboración: para obtener la ICD se parte del CDV, realizando la edición de los objetos espaciales, y el resultado es sometido a un proceso de rasterización que introduce una nueva incertidumbre en las posiciones. Por ello estos productos se evaluaron por separado.

En suma, el ámbito de la prueba se definió así: la exactitud posicional horizontal de los relieves plano, intermedio y abrupto de los CDV y las ICD correspondientes a las claves editadas de 2007 a 2011. Este ámbito consta de seis resultados, uno por cada tipo de relieve en cada producto.

Tabla 1

Ámbito de evaluación de la prueba de exactitud posicional (EP)

\begin{tabular}{|c|c|c|c|}
\hline \multirow{6}{*}{$\begin{array}{l}\text { Edición } \\
\text { 2007-2011 }\end{array}$} & Relieve plano & Rasgos geográficos & Resultado EP \\
\hline & Relieve intermedio & Rasgos geográficos & Resultado EP \\
\hline & Relieve abrupto & Rasgos geográficos & Resultado EP \\
\hline & Relieve plano & Rasgos geográficos & Resultado EP \\
\hline & Relieve intermedio & Rasgos geográficos & Resultado EP \\
\hline & Relieve abrupto & Rasgos geográficos & Resultado EP \\
\hline
\end{tabular}

Fuente: Elaboración propia.

Cada uno de estos resultados será aplicable únicamente a la parte del ámbito que le corresponde.

Respecto a cómo evaluar este ámbito, se optó por el método de evaluación clasificado por la ISO como directo-externo, ${ }^{3}$ el cual implica realizar una inspección directa de los elementos del producto y emplear como referencia datos externos (independientes) de éste.

En el caso de una prueba posicional con este método, los datos de referencia deben ser más exactos que el producto que se evalúa... los mejores disponibles. Para esta prueba se emplearon ortofotos digitales escala 1:10,000 elaboradas por el INEGI.

\section{Determinación de la muestra}

Como suele hacerse en una prueba posicional, la inspección fue de tipo muestral. La muestra se determinó por etapas. Primero se calculó la cantidad de claves 50 mil 


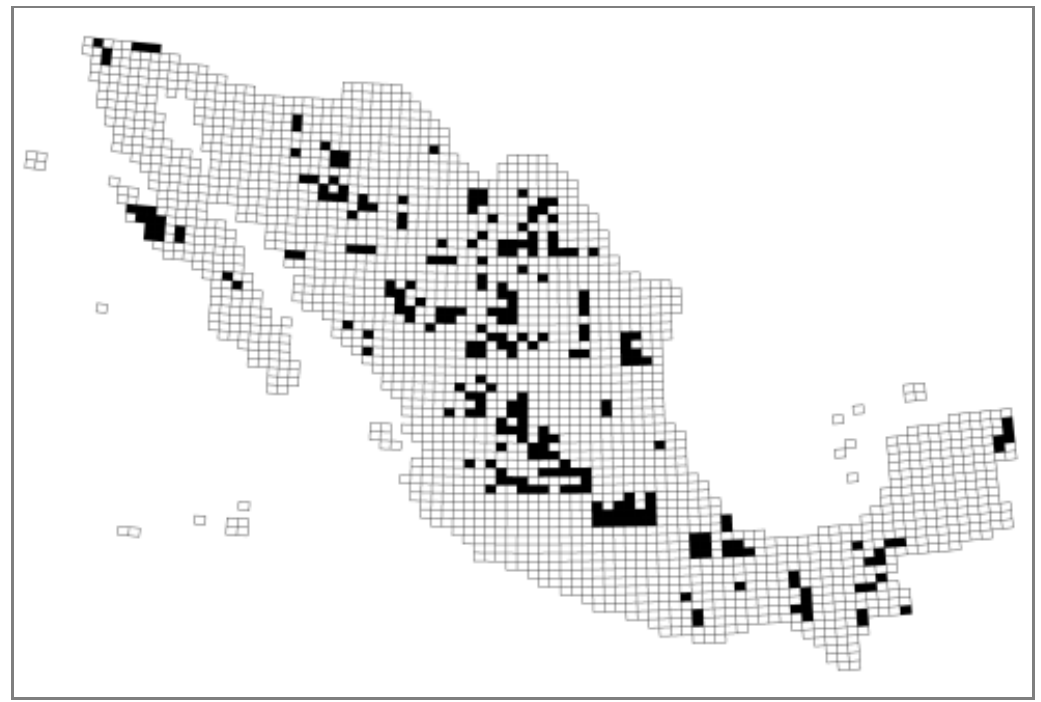

Ámbito de evaluación: 254 claves

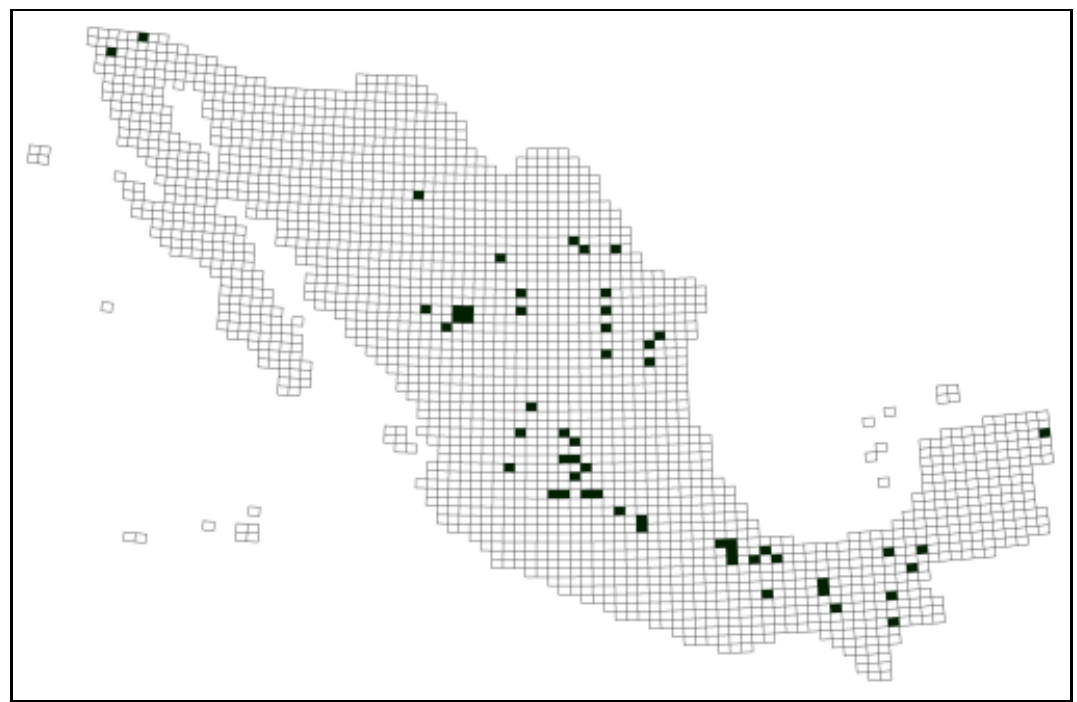

Muestra: 55 claves

Figura 2. Distribución espacial de la muestra. 
que se revisarían de cada estrato de relieve. El área de Marcos y Muestreo de la Dirección General de Estadísticas Económicas del INEGI determinó los tamaños muestrales, empleando resultados de pruebas piloto realizadas expresamente en cada uno de los tipos de relieve. El tamaño total de la muestra, incluyendo los tres estratos de relieve, fue de 55 claves (véase Tabla 2).

Habiéndose determinado cuántas claves 50 mil revisar, se procedió a decidir cuáles. Esto se hizo en cada tipo de relieve procurando la mejor distribución espacial posible, lo cual es recomendado por estándares internacionales como NSSDA. ${ }^{4}$ Es muy importante aclarar que la mejor distribución espacial en este nivel (claves 50 mil) estuvo condicionada por la cobertura de ortofotos digitales escala 1:10,000, que como ya se dijo, fuerondesignadas como datos de referencia. La distribución se muestra en la Figura 2.

El tercer aspecto en la definición de la muestra consistió en determinar la cantidad de los puntos de revisión, que los expertos consideran fundamental para lograr estimaciones estadísticas confiables. Estándares como NSSDA señalan un mínimo de 20 puntos, pero no aclaran si son para todo el producto o para cada conjunto o división del mismo. La norma STANAG, ${ }^{5}$ por su parte, recomienda un mínimo de 167 puntos. Dado que la exactitud se calcularía por separado para cada tipo de relieve, se decidió colocar 20 puntos por cada clave 50 mil, con excepción de dos claves de tipo abrupto donde se colocaron 25 (la razón se explicará más adelante). De esta manera se obtuvieron totales de puntos por tipo de relieve que cumplen holgadamente con la recomendación de STANAG (véase Tabla 2).

Tabla 2

Totales y muestras por estrato de relieve

\begin{tabular}{cccc}
\hline Estrato de relieve & Total de claves & Claves de la muestra & Total de puntos \\
\hline Plano & 91 & 23 & 480 \\
Intermedio & 99 & 13 & 240 \\
Abrupto & 64 & 19 & 390 \\
\hline Totales & 254 & 55 & 1,110 \\
\hline
\end{tabular}

Fuente: Elaboración propia.

\section{Principales criterios de la prueba y su realización}

Ya determinada la cantidad de puntos por cada clave 50 mil, se decidió su ubicación procurando nuevamente una homogénea distribución espacial, pero cuidando 


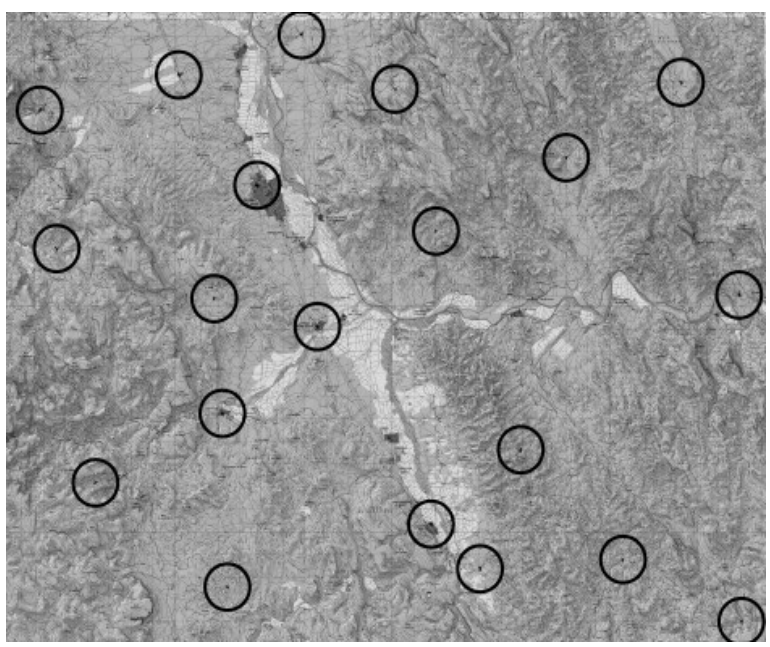

a) Abrupto

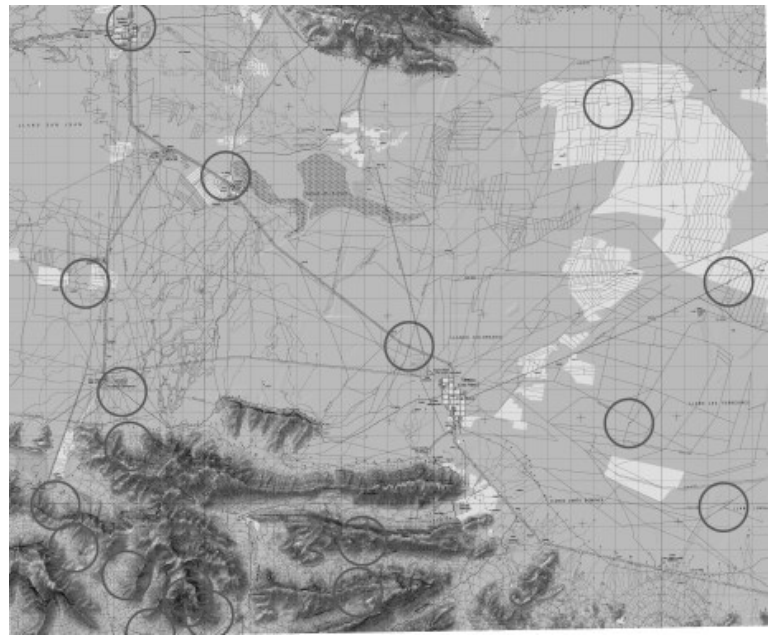

b) Intermedio

Figura 3. Disitrbución de puntos: a) estrato abrupto, con la mayoría o todos los puntos en la zona accidentada; en este caso, por la uniformidad del relieve la distribución pudo ser geométricamente regular; b) estrato intermedio, con una cantidad de puntos aproximadamente igual en zonas plana y accidentada; es una distribución espacial adecuada, aunque geométricamente pareciera no serlo. 

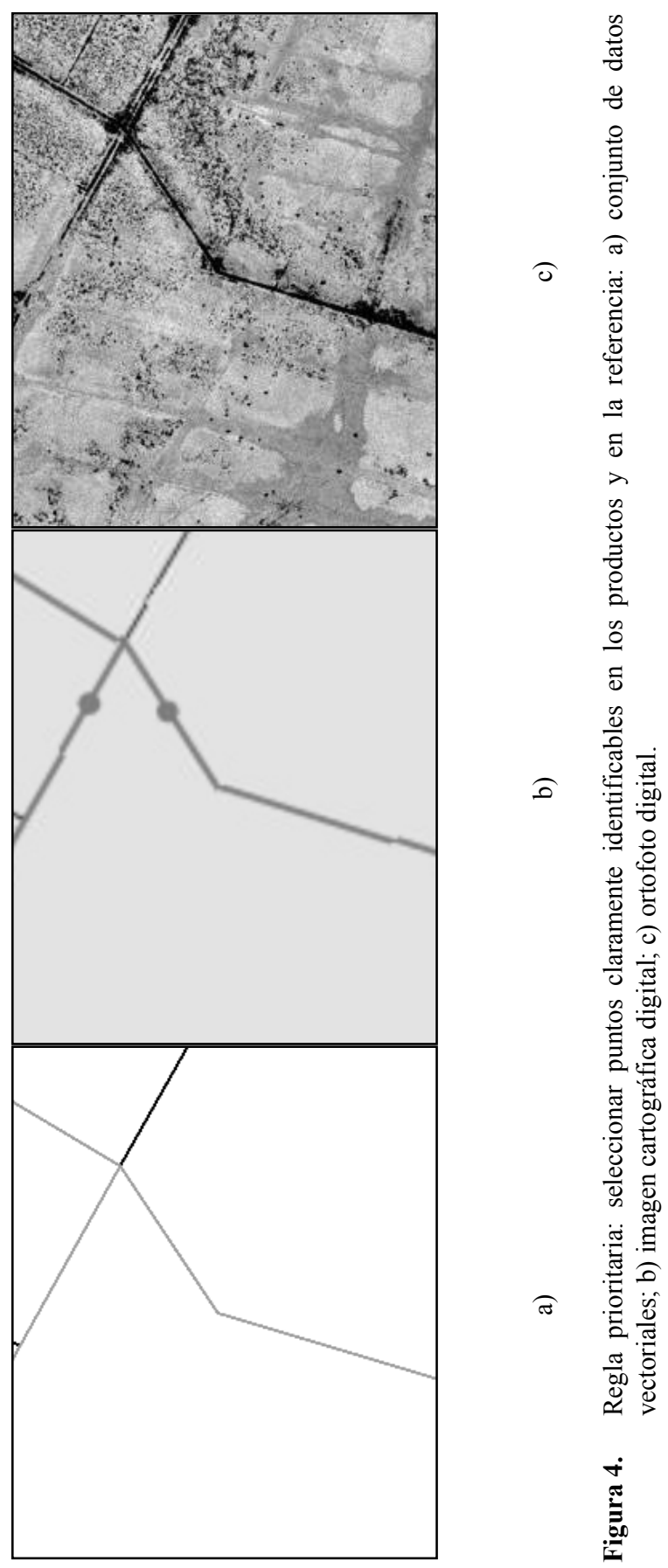
además que fuera congruente con el tipo de relieve en el cual se le clasificó. Por ejemplo, en una clave cuyo relieve fue clasificado como tipo abrupto se debía colocar la mayor cantidad de puntos en la zona accidentada y menos en la zona más plana; en los casos de relieve tipo intermedio, la cantidad de puntos en zona accidentada y plana debía ser similar (véase Figura 3).

Ahora bien, la distribución de los puntos quedó siempre condicionada por la premisa más importante en una prueba posicional: ubicar los puntos en sitios claramente identificables tanto en los productos evaluados como en la referencia; rasgos con suficiente claridad y donde no hubiera duda de que el sitio marcado era el mismo en los tres productos (Figura 4). Generalmente se trató de intersecciones o esquinas bien definidas de rasgos lineales (caminos, calles, linderos, rasgos hidrográficos, etc.) y eventualmente de elementos puntuales como construcciones claramente identificadas.

Cuando la falta de rasgos bien definidos impidió una distribución espacial adecuada de los 20 puntos, a manera de compensación se incrementó su cantidad. Esto sucedió en dos claves 50 mil del tipo abrupto. Son los casos con 25 puntos de los que se habló anteriormente. En cada una de las otras 53 claves de la muestra se colocaron 20 puntos.

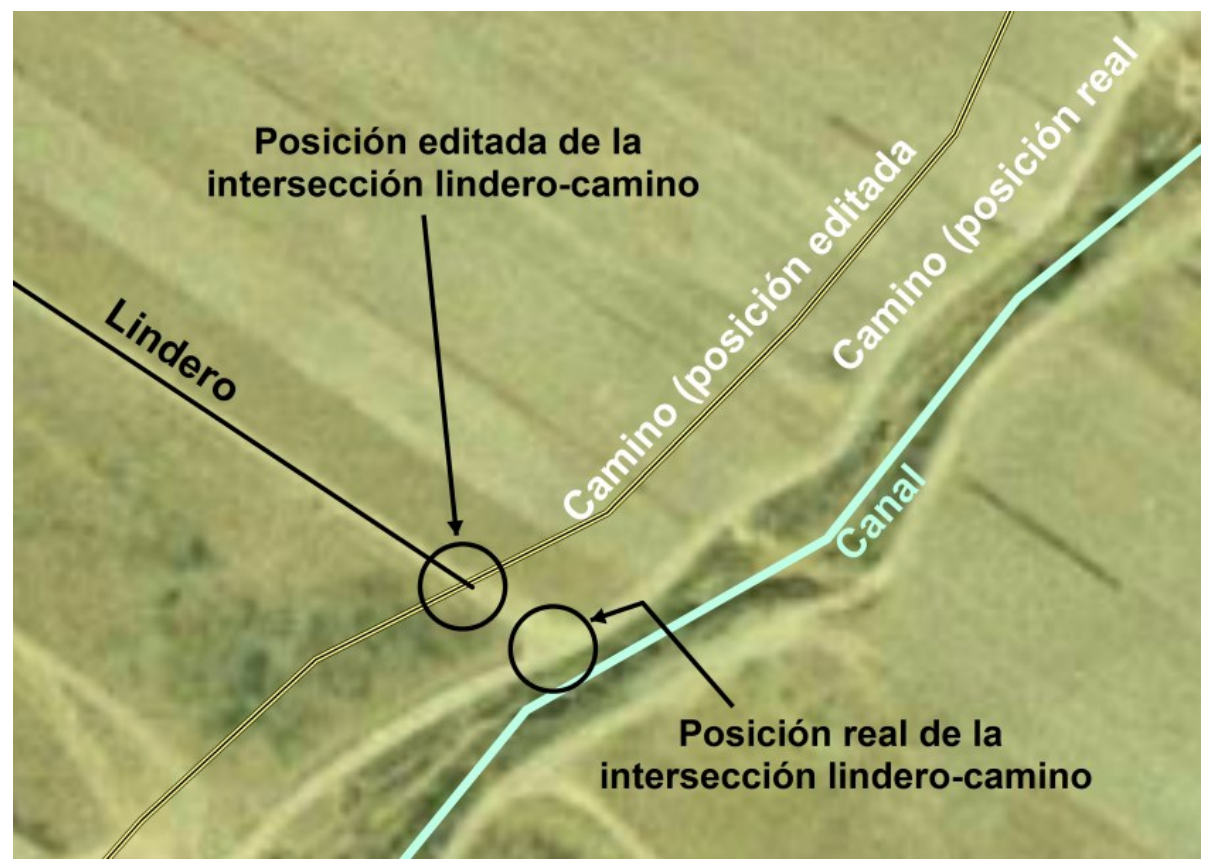

Figura 5. Los elementos desplazados por edición cartográfica no se consideraron para ubicar puntos de revisión. 

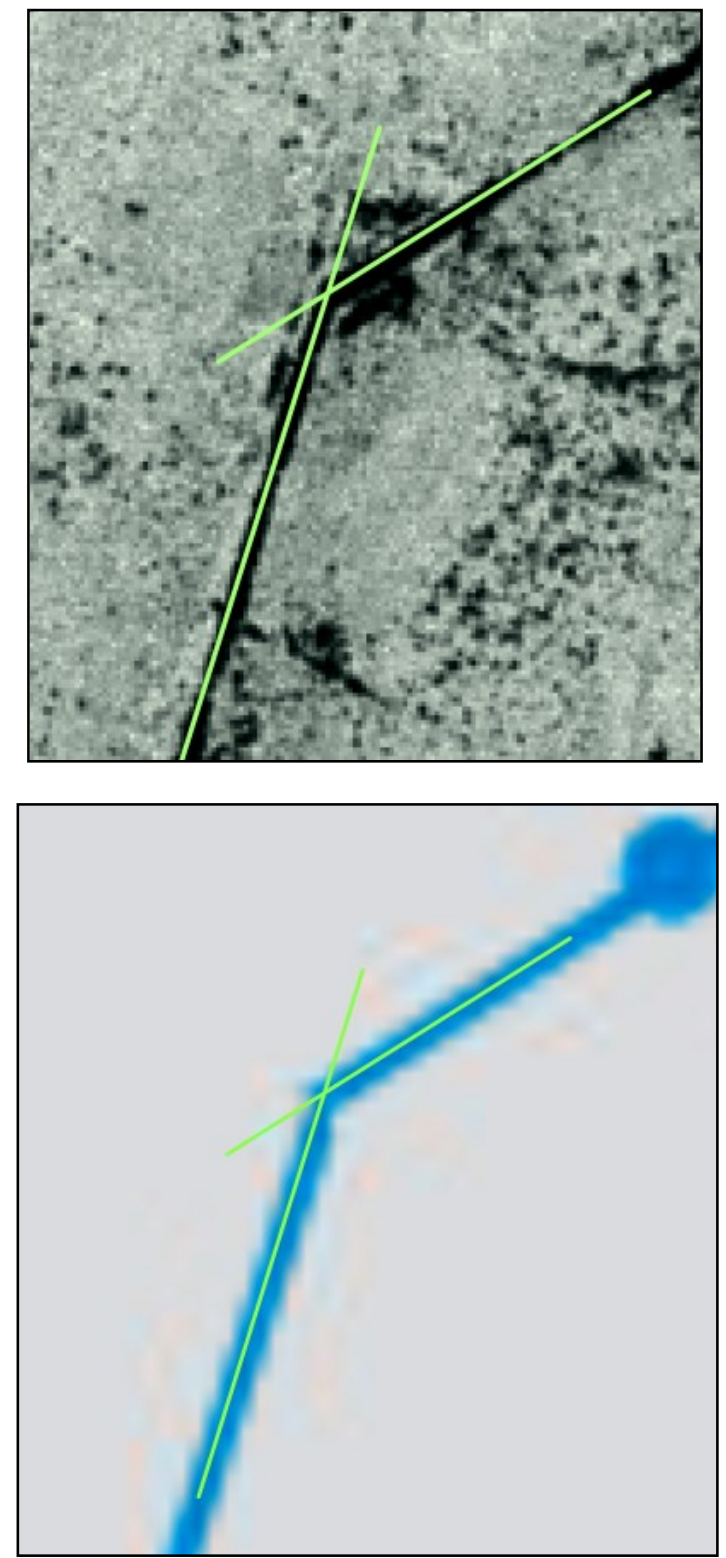

Figura 4. Líneas centrales auxiliares para colocar los puntos de revisión en el sitio correcto. Arriba: ortofoto, abajo: imagen cartográfica digital. 
Otro criterio adoptado fue no emplear rasgos desplazados por edición (Figura 5). El procedimiento de extracción vectorial, con el cual se obtienen los CDV, ubica los rasgos en su ubicación real; sin embargo, para obtener la ICD se realiza un proceso de edición, que entre otras cosas, desplaza, por razones de claridad, elementos que resultarían sobrepuestos al asignarles su simbología (como los casos de proximidad de caminos, canales, vías férreas, líneas telefónicas, etc.). Como tales casos son inherentes a cualquier producto cartográfico, no hablan realmente de su exactitud y arrojan errores atípicos (outliers) que contaminan los resultados. Lo adecuado es incluir en la información de calidad del producto las advertencias necesarias respecto al desplazamiento de objetos por razones de edición.

Por otro lado, se tomó en cuenta que en la digitalización para obtener los CDV los rasgos lineales son extraídos trazando una línea por el centro, y tales líneas son luego la base para la edición cartográfica de la cual se obtiene la ICD. Así, en la prueba, tanto en la ortofoto como en la ICD se trazaron líneas centrales auxiliares en los rasgos lineales elegidos (Figura 6) y el punto se colocó en su intersección. Con esto se logró la certeza de identificar el mismo sitio tanto en los productos como en la referencia.

Para minimizar el error humano se trabajó en un programa de SIG, en el cual los puntos fueron colocados automáticamente en las intersecciones de las líneas centrales.

Se creó un proyecto en SIG para cada una de las 55 claves 50 mil que integraron la muestra. Concluido el trabajo con cada uno, fue sometido a un control de calidad para verificar el cumplimiento de los criterios establecidos para la prueba, como los anteriormente explicados.

Como resultado, de cada uno de los 1,110 puntos se obtuvieron:

- Coordenadas X y Y en el CDV

- Coordenadas X y Y en la ICD

- Coordenadas X y Y en la referencia (ortofotos)

Disponiendo de estos valores se pudo realizar un tratamiento estadístico para calcular la exactitud.

\section{Procesamiento de resultados}

Por medios automatizados se calculó, para cada punto de revisión (tanto en la ICD como en el CDV), la diferencia entre las coordenadas en el producto y las coordenadas en la referencia, obteniéndose el valor denominado error de coordenada. De cada uno de los 1,110 puntos se obtuvieron cuatro valores de error de coordenada: de $\mathrm{X}$ y de $\mathrm{Y}$ en el CDV y de X y de Y en la ICD. 
Contra lo que podría pensarse, tener los valores de error no significa proceder de inmediato al cálculo de la exactitud. Antes se deben efectuar algunos tratamientos y pruebas de tipo estadístico.

Primero era necesario detectar los valores atípicos (outliers), que son aquéllos de magnitud que se desvía significativamente del resto de los datos. Se les considera casos que "distorsionan y reducen la información contenida en la fuente de datos". ${ }^{6}$ Para su detección se realizó la prueba K Sigmas, y los puntos donde se detectaron atipicidades fueron excluidos del cómputo final. Desde luego, tal exclusión obedece a la lógica estadística, no a la intención de ocultar errores altos. Éstos deben ser analizados para identificar el error incidental que los provocó y corregirlos.

La Tabla 3 muestra los resultados de la prueba K Sigmas. Tanto en los CDV como en la ICD se detectaron valores atípicos en 14 puntos. $^{7}$

Tabla 3

Valores atípicos por producto y estrato de relieve

\begin{tabular}{ccccc}
\hline $\begin{array}{c}\text { Puntos } \\
\text { originales }\end{array}$ & $\begin{array}{c}C D V \\
\text { Puntos } \\
\text { con atipicos }\end{array}$ & $\begin{array}{c}\text { ICD } \\
\text { Puntos } \\
\text { con atipicos }\end{array}$ & $\begin{array}{c}\text { CDV } \\
\text { Puntos } \\
\text { definitivos }\end{array}$ & $\begin{array}{c}\text { ICD } \\
\text { Puntos } \\
\text { definitivos }\end{array}$ \\
\hline 480 & 5 & 3 & 475 & 477 \\
240 & 3 & 5 & 237 & 235 \\
390 & 6 & 6 & 384 & 384 \\
\hline 1,110 & 14 & 14 & 1,096 & 1,096 \\
\hline
\end{tabular}

Fuente: Elaboración propia.

En suma, tanto para el CDV como para la ICD se descartaron 14 puntos, así que el total empleado para el cómputo final fue de 1,096. Dado que el mínimo recomendado por la norma STANAG es de 167 , la cantidad disponible siguió siendo holgadamente suficiente.

Por otro lado, la elección del indicador para calcular resultados requirió otras pruebas estadísticas. Una de las referencias más usadas para medir la exactitud posicional es el National Stantard for Spatial Data Accuracy (NSSDA), desarrollado por el Federal Geographic Data Committee de Estados Unidos. Sin embargo, el mismo establece para su aplicación ciertas condiciones que deben cumplir los datos, como una distribución normal y ausencia de sesgos. Si no se cumplen el resultado

6 Ariza, Fundamentos de evaluación de la calidad de la información geográfica, 2014, p. 146.

7 Por coincidencia resultó la misma cantidad de atípicos detectados en cada producto. No son exactamente los mismos puntos. Por ejemplo, algunos puntos con atipicidad en el CDV no la presentaron en la ICD. 
que se calcule será erróneo. Por ello se aplicaron a los datos las pruebas Kolmogorov-Smirnov para verificar la normalidad, y T de Student para la detección de sesgos. Las pruebas se aplicaron para cada una de las series de valores de error: en cada componente ( $\mathrm{X}$ y Y) de cada producto (CDV e ICD) en cada uno de los tres estratos de relieve. En suma, fueron 12 pruebas de normalidad y otras 12 de sesgos.

Los resultados mostraron que no todos los componentes tenían distribución normal, y en algunos se detectaron sesgos que rebasaban el límite aceptable. Para conocer las causas se requeriría una investigación detallada; pero de manera inmediata los resultados indicaron que aplicar la fórmula propuesta por NSSDA no habría sido técnicamente válido, ni congruente con los principios de honestidad que deben guiar todo empeño para evaluar y declarar la calidad.

Por ello, para el cómputo final se optó por aplicar la raíz del error medio cuadrático (EMC), índice que figura entre las medidas de calidad aceptadas por la Organización Internacional de Normalización (ISO), [Norma técnica 19157 - Geographic Information - Data Quality, 2013]. Los resultados por cada estrato de relieve y producto se muestran en la tabla 4.

Tabla 4

Resultados de la prueba de exactitud posicional en productos topográficos digitales escala 1:50,000

\begin{tabular}{|c|c|c|c|c|c|c|}
\hline \multirow{2}{*}{$\begin{array}{l}\text { Estrato } \\
\text { de } \\
\text { relieve }\end{array}$} & \multirow[b]{2}{*}{ Producto } & \multirow[b]{2}{*}{ Componente } & & \multirow{2}{*}{$\begin{array}{c}\text { Puntos } \\
\text { originales } \\
\text { definitivos }\end{array}$} & \multicolumn{2}{|c|}{ Error medio cuadrático** } \\
\hline & & & & & componente & Planimétrico $* * *$ \\
\hline \multirow{4}{*}{ Plano } & $\mathrm{CDV}$ & Coordenada X & 480 & 475 & $4.9080 \mathrm{~m}$ & $6.515 \mathrm{~m}$ \\
\hline & $\mathrm{CDV}$ & Coordenada Y & 480 & 475 & $4.2840 \mathrm{~m}$ & $6.515 \mathrm{~m}$ \\
\hline & $\overline{\mathrm{ICD}}$ & Coordenada X & 480 & 477 & $5.4529 \mathrm{~m}$ & $7.387 \mathrm{~m}$ \\
\hline & ICD & Coordenada Y & 480 & 477 & $4.9837 \mathrm{~m}$ & $7.387 \mathrm{~m}$ \\
\hline \multirow{4}{*}{$\begin{array}{l}\text { Inter- } \\
\text { medio }\end{array}$} & $\mathrm{CDV}$ & Coordenada X & 240 & 237 & $3.7498 \mathrm{~m}$ & $4.971 \mathrm{~m}$ \\
\hline & $\mathrm{CDV}$ & Coordenada Y & 240 & 237 & $3.2635 \mathrm{~m}$ & $4.971 \mathrm{~m}$ \\
\hline & ICD & Coordenada X & 240 & 235 & $5.0699 \mathrm{~m}$ & $6.488 \mathrm{~m}$ \\
\hline & ICD & Coordenada Y & 240 & 235 & $4.0488 \mathrm{~m}$ & $6.488 \mathrm{~m}$ \\
\hline \multirow{4}{*}{$\begin{array}{l}\text { Abrup- } \\
\text { to }\end{array}$} & $\mathrm{CDV}$ & Coordenada X & 390 & 384 & $5.8290 \mathrm{~m}$ & $7.885 \mathrm{~m}$ \\
\hline & CDV & Coordenada Y & 390 & 384 & $5.3096 \mathrm{~m}$ & $7.885 \mathrm{~m}$ \\
\hline & $\overline{\mathrm{ICD}}$ & Coordenada X & 390 & 384 & $6.5334 \mathrm{~m}$ & $8.986 \mathrm{~m}$ \\
\hline & ICD & Coordenada Y & 390 & 384 & $6.1691 \mathrm{~m}$ & $8.986 \mathrm{~m}$ \\
\hline
\end{tabular}

* Los restantes tras eliminar los que presentaron valor atípico.

** Calculado con los puntos definitivos.

*** Declaración en metadatos.

Fuente: Elaboración propia. 
Como se explicó antes, se asumió una exactitud diferente por tipo de relieve y de producto, y los resultados tienden a confirmar en términos generales esta hipótesis. El error más bajo se presentó en el CDV y en el relieve plano. En contraste, el error más alto - la menor exactitud - correspondió a la ICD con relieve abrupto; es decir donde se combinan la topografía que ofrece más dificultades para la corrección fotogramétrica y el producto cuyo proceso de elaboración incluye edición, simbolización y posterior rasterización. Del relieve intermedio se esperarían entonces valores que correspondieran con una tendencia progresiva, pero los resultados no coinciden completamente con ello. No se pueden, sin embargo, adelantar explicaciones sin un estudio detallado. Esta evaluación es apenas el inicio de varias tareas a las que se enfrenta todo productor de información geográfica que afronta el reto de conocer la calidad de sus productos.

Es necesario resaltar que la estrategia de tratamiento e integración de resultados antes reseñada se realizó tomando en cuenta valiosos consejos del doctor Francisco Javier Ariza, experto en calidad de la Universidad de Jaén, España.

\section{Conclusiones}

La evaluación de la calidad tiene dos grandes virtudes, como aclara ISO [Norma 19157]. Por un lado, permite a los usuarios determinar en qué medida los datos espaciales cubren sus requerimientos; la separación de cálculos por clase de producto y estrato de relieve tiene la virtud de ofrecer datos que los usuarios pueden considerar y analizar con sus perspectivas. Por otro lado, permite al productor saber qué tanto sus datos cumplen con las especificaciones establecidas para ellos. Es un primer paso indispensable para establecer en el futuro los requisitos de calidad que deben cumplir los productos, y más importante, las especificaciones de producción que garanticen ese cumplimiento. En suma, lo que se conoce como aseguramiento de la calidad.

De esta manera se informa de la confiabilidad técnica de los productos geográficos. Más que decirle cómo usarlos, la misión del productor es proporcionar al usuario potencial la información necesaria para que estime si un determinado producto es suficiente para sus requerimientos. Cuando se declaren los resultados de la prueba antes reseñada, cada usuario podrá determinar cuál es la aptitud de uso que para él posee, en el aspecto posicional, la información topográfica escala 1:50,000 del INEGI. Lo anterior significa que el trabajo no concluye sino hasta que en los metadatos del producto se incluya la información de calidad. Ése es el fin último y necesario. De esta manera se hace evidente que en la institución se ha adoptado la noción de fitness for use.

Pero con eso no se ha hecho más que iniciar un proceso de años. Es importante avanzar en esta etapa de evaluación y declaración de la calidad; pero este avance debe hacerse con un rumbo claro, y éste debe ser hacia el aseguramiento de la cali- 
dad, estrechando la comunicación con los usuarios para que dicha calidad sea tal que cubra lo mejor posible las necesidades de información de la sociedad actual. Éstas deben ser la base para establecer los requisitos de calidad. De esa manera se asegura que los productos geográficos serán útiles, justificando plenamente los recursos invertidos en su elaboración.

Como bien se ha dicho, la calidad es ineludible, aunque cueste. Y también lo es la perspectiva de incrementarla paulatinamente, de manera que su utilidad, su capacidad de satisfacer necesidades específicas, sea cada vez más incluyente. La noción de aptitud para el uso no es una llegada, sino un punto de partida.

\section{Bibliografía}

Ariza, F.J. (ed.) 2014. Fundamentos de evaluación de la calidad de la información geográfica, Universidad de Jaén, España.

Conalton, R. y Green, K. (2009). Assessing the accuracy of remotely sensed data, CRC Press, USA.

Federal Geographic Data Committee (FGDC) (1998). National Standard for Spatial Data Accuracy, USA.

International Organization for Standarization (ISO) (2013). International Standard 19157, Geographic Information-Data Quality, Suiza.

North Atlantic Treaty Organization (NATO), Standarization Agency (2002). Standarization Agreement (STANAG), Bruselas. 